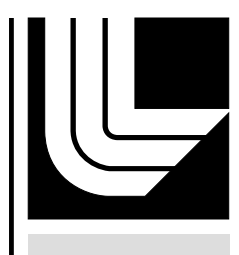

LAW RENCE LIVERMORE N A T IO N A L LABORATORY

EEMP Summary for NIF shot:

N101004-002-999

J. Kimbrough

October 13, 2010 
This document was prepared as an account of work sponsored by an agency of the United States government. Neither the United States government nor Lawrence Livermore National Security, LLC, nor any of their employees makes any warranty, expressed or implied, or assumes any legal liability or responsibility for the accuracy, completeness, or usefulness of any information, apparatus, product, or process disclosed, or represents that its use would not infringe privately owned rights. Reference herein to any specific commercial product, process, or service by trade name, trademark, manufacturer, or otherwise does not necessarily constitute or imply its endorsement, recommendation, or favoring by the United States government or Lawrence Livermore National Security, LLC. The views and opinions of authors expressed herein do not necessarily state or reflect those of the United States government or Lawrence Livermore National Security, LLC, and shall not be used for advertising or product endorsement purposes.

This work performed under the auspices of the U.S. Department of Energy by Lawrence Livermore National Laboratory under Contract DE-AC52-07NA27344. 


\section{EEMP summary for NIF shot: N101004-002-999}

Preliminary

A.Throop 10/05 08:30

\begin{tabular}{|l|}
\hline Non-damaging EMP fields \\
(no damage reports). \\
Low EMP. Similar to, \\
slightly lower than, similar \\
N100821 shot below.
\end{tabular}

Internal EMP peak field*: $\sim 0.8 \mathrm{kV} / \mathrm{m}^{* *}$

*at $R=4.0 \mathrm{~m}$ from TCC, for B270 Bdot sensor ( $1 \mathrm{GHz}$ bandwidth). Fields may vary $\sim 1 / R^{2}$, so fields at $R=1 \mathrm{~m}$ could be $\sim 16 \mathrm{x}$ larger than

** Qlook2 w/ spectrum analysis
Shot ID: IT_0_Symcap_S03t Laser: $1.06 \mathrm{MJ}$; Hahn 3ns; 188b, 47q. Target: Cold gas Hohl: Au, 359T He4. Cap: $6 \% \mathrm{D}+70 \% \mathrm{He}, 2992 \mathrm{~T}=3.93 \mathrm{~atm}$ Notes: Neutron Yield NA.

\section{COMPARISON: N100821, 1.04} MJ, IT_0_Symcap_s07h
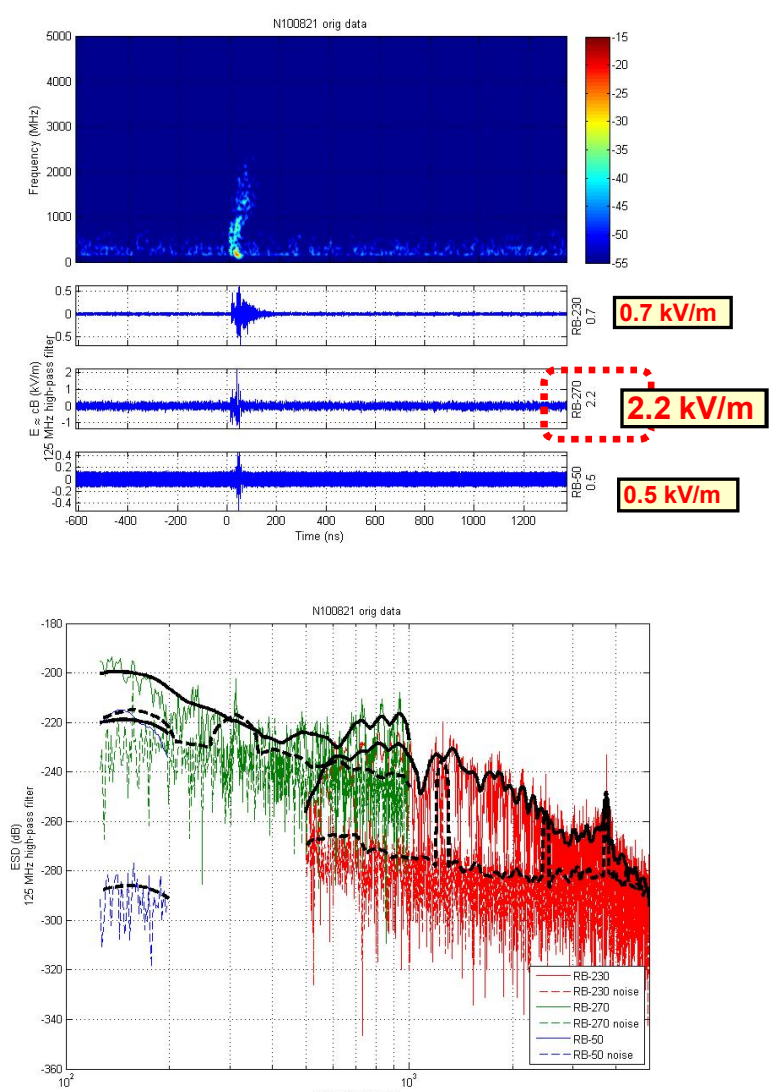

Frequency (MHz)

\begin{tabular}{c}
$\frac{\text { THIS SHOT : N101004-002, } 1.06 \mathrm{MJ},}{\text { IT_0_Symcap_S03t }}$ \\
\hline
\end{tabular}
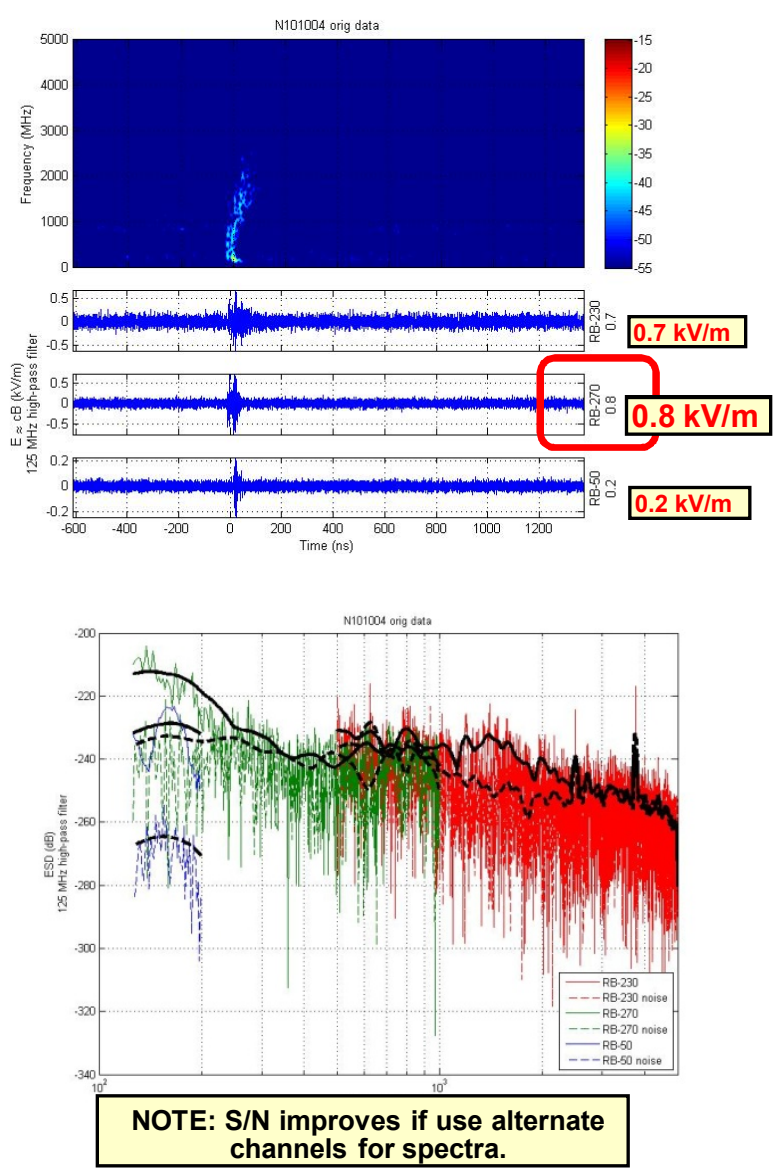

Page 1

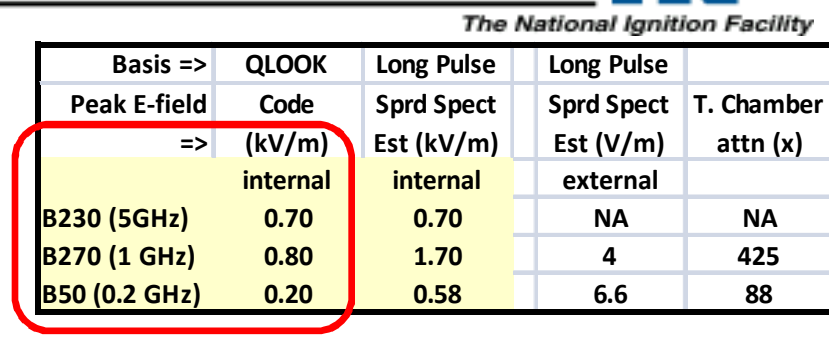

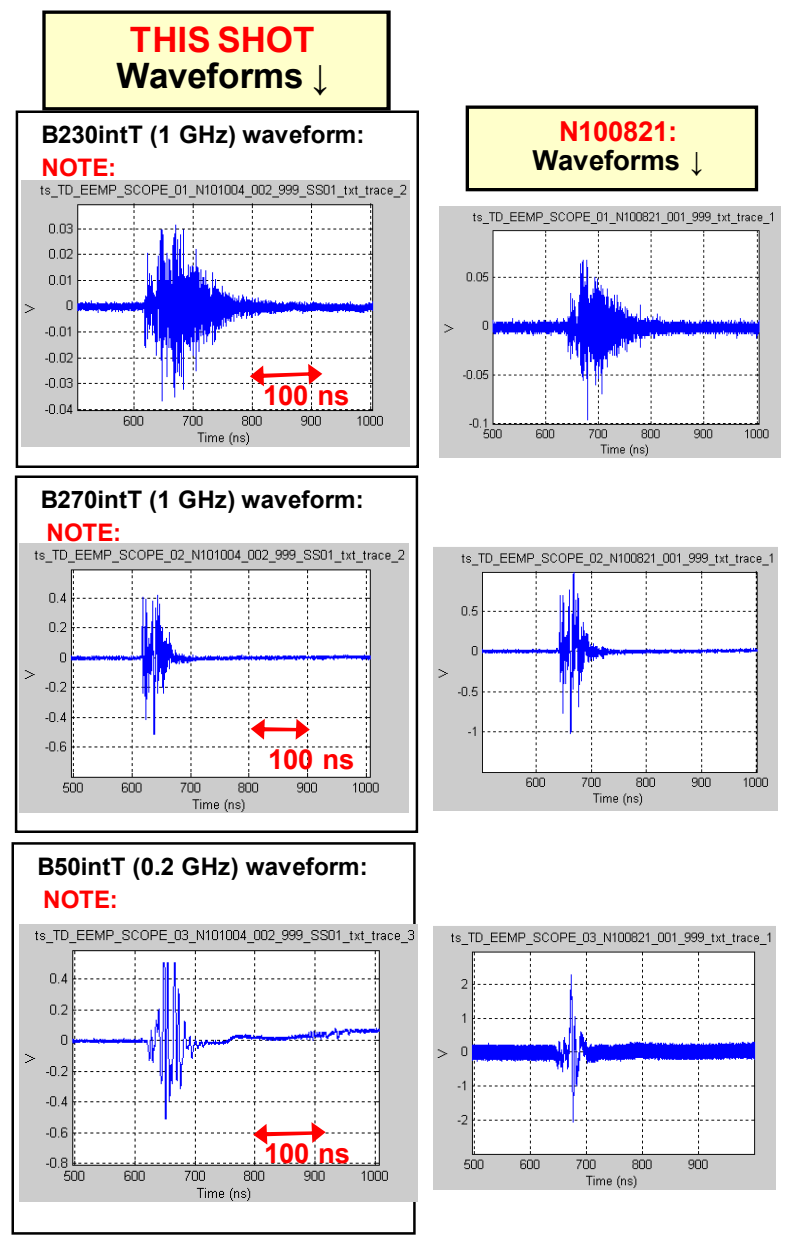

EEMP summary chart N101004-002 